\title{
ON THE DIMENSION OF VEBLEN-WEDDERBURN SYSTEMS
}

\author{
by CARLTON J. MAXSON
}

(Received 11 March, 1969)

1. Introduction. In [1, p. 97], Bruck and Bose ask the question " Has every (right) Veblen-Wedderburn system finite dimension over its left operator skew-field?" It is the purpose of this note to show that, in general, this question has a negative answer.

We recall that, in [1], the left operator skew-field of a Veblen-Wedderburn system $\langle R,+,$. is defined to be the subsystem $\langle F,+,$.$\rangle consisting of those elements x \in R$ satisfying, for all $a, b \in R$,

(i) $x \cdot(a+b)=x \cdot a+x \cdot b$,

(ii) $x \cdot(a \cdot b)=(x \cdot a) \cdot b$

The Veblen-Wedderburn systems considered in this paper will be (right) near-fields $\langle F,+,$.$\rangle with the additional property$

$(P)$ for all $a, b, c \in F, a \neq b$, there exists one and only one element $x \in F$ such that $x a=x b+c$.

We recall that a near-field is an algebraic system $\langle F,+,$.$\rangle such that +$ and . are associative binary operations on $F,\langle F,+\rangle$ is a group with identity 0 (say), $\langle F-\{0\},$.$\rangle is a group and$ $(a+b) . c=a . c+b . c$ for all $a, b, c \in F$. As usual $a . c$ will be written $a c$ and the multiplicative identity denoted by 1 .

The near-fields satisfying property $(P)$ are called planar by Zemmer [4] and projective by Kerby [2].

The kern of a near-field $\langle F,+,$.$\rangle is defined to be the set K(F)=\{a \in F \mid a(b+c)=a b+a c$ for all $b, c \in F\} .\langle K(F),+,$.$\rangle is a subskew-field of F$ and $\langle F,+\rangle$ is a (left) vector space over $K(F)$. In particular, the kern of $F$ is the left operator skew-field of $F$ (considering $F$ as a Veblen-Wedderburn system). Moreover, if the dimension of $\langle F,+\rangle$ over $K(F)$ (i.e. $[F: K(F)]$ ) is finite, then $\langle F,+,$.$\rangle is a planar near-field (see [2] and [4]).$

Both Kerby and Zemmer give examples of near-fields not satisfying property $(P)$. Kerby also gives an example of an infinite near-field satisfying $(P)$. We use the methods of Kerby to construct infinite planar near-fields (hence Veblen-Wedderburn systems) which are infinite dimensional over $K(F)$ (i.e., over their left operator skew-fields).

In our construction of infinite planar near-fields, we use the concept of " coupling map " defined in [3]. For the sake of completeness, we give this definition.

Definition. Let $\langle R,+,$.$\rangle be a ring and \operatorname{End}_{0} R$ be the semigroup of ring endomorphisms of $R$ with $0_{R}$ adjoined. A function $\phi: R \rightarrow \operatorname{End}_{0} R\left(a \rightarrow \phi_{a}\right)$ is said to be a coupling map of $R$ if $\phi_{0}=0_{R}$ and $\phi_{a} \circ \phi_{b}=\phi_{a \phi_{b} \cdot b}$, for all $a, b \in R$.

2. Results. Let $H$ be a field and $T$ an arbitrary but fixed automorphism of $H, T \neq I_{H}$. $T$ induces an automorphism $T^{*}$ on $H((x))$, the field of formal power series over $H$; that is, for $\alpha=\sum_{h}^{\infty} \alpha_{i} x^{i} \in H((x)), \alpha T^{*}=\sum_{h}^{\infty}\left(\alpha_{i}\right) T x^{i}$. The mapping $\phi: H((x)) \rightarrow \operatorname{End}_{0}\langle H((x)),+,$. 
given by

$$
\alpha \phi=\left\{\begin{aligned}
\alpha\left(T^{*}\right)^{\delta(\alpha)}, & \alpha \neq 0, \\
0, & \alpha=0,
\end{aligned}\right.
$$

where $\delta(\alpha)=$ Ord $\alpha$ (= smallest index for which $\alpha_{i} \neq 0$ ), is a coupling map for $H((x))$ and therefore (see [3], p. 6) $\langle H((x)),+, \circ\rangle$ is a near-field. We recall that the multiplication $\circ$ is given by

$$
\alpha \circ \beta=\left\{\begin{array}{cl}
0, & \beta=0, \\
\alpha\left(T^{*}\right)^{\delta(\beta)} . \beta, & \beta \neq 0 .
\end{array}\right.
$$

Thus, if $\alpha=\sum_{r}^{\infty} \alpha_{i} x^{i}$ and $0 \neq \beta=\sum_{i}^{\infty} \beta_{j} x^{j}$, then $\alpha_{\circ} \beta=\sum_{r}^{\infty}\left(\alpha_{i}\right) T^{\delta(\beta)} x^{i} \cdot \sum_{t}^{\infty} \beta_{j} x^{j}$.

Kerby [2] has shown that $\langle H((x)),+, 0\rangle$ is a near-field with property $(P)$.

In particular, let $H$ be the field $k(x)$ of rational functions in one indeterminate over a field $k$ of characteristic zero. Let $T: k(x) \rightarrow k(x)$ be the automorphism of $k(x)$ given by $x \rightarrow x+1$. We denote the coupled near-field $\langle k(x)((t)),+, 0\rangle$ by $F$. We proceed to show that $[F: K(F)]$ is not finite.

LEMMA Let $\alpha=\sum_{h}^{\infty} \alpha_{i} t^{i} \in F$; then $\alpha \in K(F)$ if and only if $\alpha_{i} T=\alpha_{i}$ for all $i$.

Proof. If $\alpha \in K(F)$, then $\alpha \circ(1+t)=\alpha \circ 1+\alpha \circ t=\alpha+\alpha \circ t$. Hence $\alpha \circ(1+t)=\alpha \cdot(1+t)=$ $\alpha+\sum_{h}^{\infty} \alpha_{i} T t^{i+1}$, which in turn implies that $\sum_{h}^{\infty} \alpha^{i} t^{i+1}=\sum_{h}^{\infty} \alpha_{i} T t^{i+1}$. Thus $\alpha_{i}=\alpha_{i} T$ for all $i$. Conversely, if this is the case, then, for all $n \in Z, \alpha_{l} T^{n}=\alpha_{i}$. Hence $\alpha \circ \beta=\alpha . \beta$ for all $\beta \in F$, and so $\alpha \in K(F)$.

COROLlary. Let $\alpha=\sum_{h}^{\infty} \alpha_{i} t^{i} \in F$; then $\alpha \in K(F)$ if and only if $\alpha_{i} \in k$, for all $i$.

Proof. Let $q \in k(x), q=f(x) / g(x)$, where $f(x), g(x) \in k[x], g(x) \neq 0$; we may assume without loss of generality that g.c.d. $\{f(x), g(x)\}=1$. We must verify that $q T=q$ is equivalent to $q \in k$. Clearly $q \in k$ implies that $q T=q$. Conversely, $f(x) / g(x)=f(x+1) / g(x+1)$ implies that $f(x) g(x+1)=f(x+1) g(x)$. Hence $f(x) \mid f(x+1)$ and so $f(x+1)=r f(x)$, where $r \in k$. Let $f(x)=\sum_{i=0}^{\infty} a_{i} x^{i}$, where $a_{n} \neq 0$. Assume that $n \geqq 1$. From $f(x+1)=r f(x)$, by equating the coefficients of $x^{n}$ and $x^{n+1}$, one obtains

(i) $a_{n}=r a_{n}$,

(ii) $n a_{n}+a_{n-1}=r a_{n-1}$.

From (i), $r=1$, since $a_{n} \neq 0$. But then, from (ii), $n a_{n}=0$, which is a contradiction since $k$ is of characteristic zero. Hence $f(x)=a_{0} \in k$. If $a_{0}=0$, then $q \in k$. If $a_{0} \neq 0$, we obtain $g(x)=$ $g(x+1)$ and then find that $g(x)=b_{0} \in k$. Hence $q \in k$, as desired.

Since $k(x)$ is a simple transcendental extension of $k,[k(x): k]=\infty$. Let $B=\left\{b_{u} \mid \alpha \in \Lambda\right\}$ be a basis for $k(x)$ over $k$. Since $B \subseteq k(x)$, we have $B \subseteq k(x)((t))$. For any finite subset 
$\left\{b_{\alpha_{i}} \mid i=1,2, \ldots, r\right\}$ of $B$, let $0=b_{\alpha_{1}} f_{1}+b_{\alpha_{2}} f_{2}+\ldots+b_{\alpha_{r}} f_{r}$, where $f_{i} \in K(F), i=1,2, \ldots, r$. Hence $f_{i}=\sum_{h_{i}}^{\infty} a_{j}^{i} t^{j}, a_{j}^{i} \in k$. For each $j \geqq h=\min \left\{h_{i} \mid i=1,2, \ldots, r\right\}$,

$$
0=b_{\alpha_{1}} a_{j}^{1}+b_{\alpha_{2}} a_{j}^{2}+\ldots+b_{\alpha_{r}} a_{j}^{r}
$$

and since $[k(x): k]=\infty$, we have $a_{j}^{i}=0$ for $i=1,2, \ldots, r$ and all $j$. Hence $B$ is an independent set over $K(F)$ and consequently $[F: K(F)] \geqq[k(x): K(F)]=\infty$.

We have established the following

THEOREM. There exist Veblen-Wedderburn systems having infinite dimension over their left operator skew-fields.

\section{REFERENCES}

1. R. H. Bruck and R. C. Bose, The construction of translation planes from projective spaces, J. Algebra 1 (1964), 85-102.

2. W. Kerby, Projective und nicht-projective Fastkörper, Abh. Math. Sem. Univ. Hamburg, 32 (1968), 20-24.

3. C. J. Maxson, Dickson near-rings, J. Algebra 14 (1970), 152-169.

4. J. L. Zemmer, Near-fields, planar and non-planar, The Math. Student, 31 (1964), 145-150.

TEXAS A and M UNIVERSITY

College Station, Texas 77843 